\title{
A characterization of extremal graphs with no matching-cut
}

\section{Paul Bonsma}

Faculty of Electrical Engineering, Mathematics and Computer Science, University of Twente, Netherlands

\begin{abstract}
A graph is called (matching-)immune if it has no edge cut that is also a matching. Farley and Proskurowski proved that for all immune graphs $G=(V, E),|E| \geq\lceil 3(|V|-1) / 2\rceil$, and constructed a large class of immune graphs that attain this lower bound for every value of $|V(G)|$, called ABC graphs. They conjectured that every immune graph that attains this lower bound is an $\mathrm{ABC}$ graph. We present a proof of this conjecture.
\end{abstract}

Keywords: matching-cut, matching immune, extremal graphs

\section{Introduction}

All graphs in this paper are allowed to be multi-graphs. For a graph $G=(V, E)$, an edge cut $[S, \bar{S}]$ with $S \subset V$ is called a matching-cut if $[S, \bar{S}]$ is a matching. If a graph has no matching-cut, it is called (matching-)immune. Farley and Proskurowski [Farley and Proskurowski(1984)] proved the following extremal result on immune graphs:

Theorem 1 (Farley and Proskurowski) If $G=(V, E)$ is immune, then

$$
|E| \geq\lceil 3(|V|-1) / 2\rceil .
$$

In addition, they constructed a class of multi-graphs called $A B C$ graphs that have the following properties:

- $\mathrm{ABC}$ graphs are immune.

- If $G=(V, E)$ is an ABC graph, then $|E|=\lceil 3(|V|-1) / 2\rceil$.

The definition of $\mathrm{ABC}$ graphs is based on the following three operations:

- An A operation on vertex $u$ introduces vertices $v$ and $w$ and edges $u v, u w$ and $v w$.

- A B operation on edge $u v$ introduces vertices $w$ and $x$ and edges $u w, v w, u x$ and $v x$, and removes edge $u v$.

- A C operation on vertices $u$ and $v(u=v$ is allowed) introduces vertex $w$ and edges $u w$ and $v w$.

Note that the $\mathrm{C}$ operation is the only operation that can introduce parallel edges. 

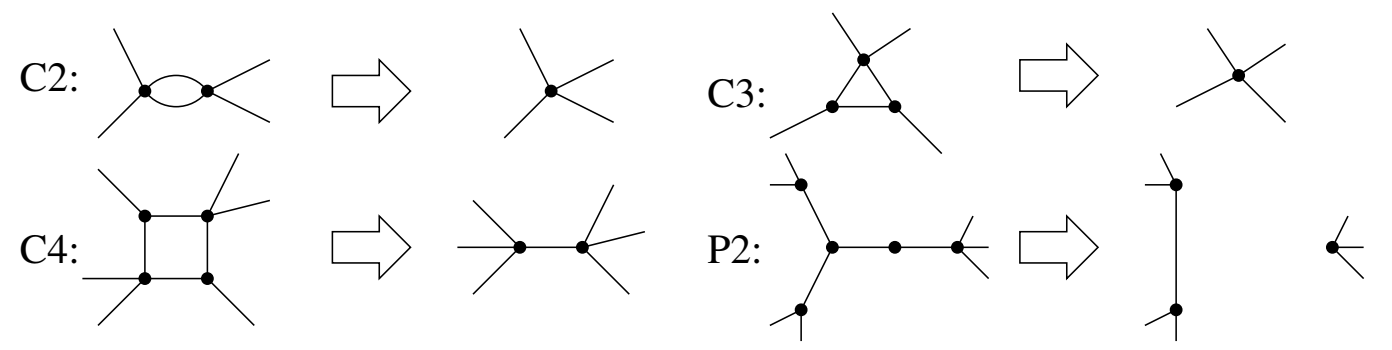

Fig. 1: The four reduction operations

Definition $1 \mathrm{An} \mathrm{ABC}$ graph is a graph that can be obtained from $K_{1}$ with a sequence of $A$ and $B$ operations and at most one $C$ operation.

A sequence of these operations that constructs a graph $G$ from a graph $H$ is called a decomposition of $G$ from $H$, or simply a decomposition of $G$ if $H=K_{1}$. For every integer $n \geq 1$, an ABC graph exists. For every integer $n \geq 1, n \neq 2$, a simple $\mathrm{ABC}$ graph exists. This shows that the lower bound from Theorem 1 is tight. This inspires the following definition:

Definition 2 A graph $G=(V, E)$ is called extremal immune if $|E|=\lceil 3(|V|-1) / 2\rceil$ and $G$ is immune.

Farley and Proskurowski stated the following conjecture:

Conjecture 2 (Farley and Proskurowski) If a graph $G$ is extremal immune, then $G$ is $A B C$.

In this paper, we sketch our proof of this conjecture, and show some of the techniques that are used. First we will go into more detail on Farley and Proskurowski's proof of Theorem 1.

\section{Contraction operations}

To prove Theorem 1, Farley and Proskurowski [Farley and Proskurowski(1984)] introduce four graph operations, named after the structure they reduce. These operations are explained in Figure 1. Below are formal definitions, which show that all of these operations can be expressed with edge deletions and contractions. $C_{2}$ stands for the cycle of length two, which is a multi-graph.

C2 Let vertices $u$ and $v$ induce a $C_{2}$ in graph $G$. The $\mathrm{C} 2$ operation consists of deleting one of the edges of this $C_{2}$ and contracting the other.

C3 Let vertices $u, v$ and $w$ induce a $C_{3}$ in $G$. The $\mathrm{C} 3$ operation consists of deleting $u v$ and contracting $v w$ and $w u$.

C4 Let vertices $u, v, w$ and $x$ induce a $C_{4}$ in $G$ such that $u w \notin E(G)$. The $\mathrm{C} 4$ operation consists of deleting $u v$ and contracting $u x$ and $v w$. Note that for one induced $C_{4}$ the $\mathrm{C} 4$ operation can be applied in two different ways.

P2 Let vertices $u$ and $v$ be neighbors in $G$ with $d(u)=3$ and $d(v)=2$. Let $v$ have another neighbor $z \neq u$, and let $u$ have another neighbor $x \neq v$. The $\mathrm{P} 2$ operation consists of deleting $u v$ and contracting $u x$ and $v z$. 
It can be checked that these four operations have the following properties:

Lemma 3 (Farley and Proskurowski) Suppose $G^{\prime}$ can be obtained from G by a C2, C3, C4 or P2 operation. Then:

- If $G$ is immune, then $G^{\prime}$ is immune.

- If $|E(G)|=\lceil 3(|V(G)|-1) / 2\rceil$, then $\left|E\left(G^{\prime}\right)\right| \leq\left\lceil 3\left(\left|V\left(G^{\prime}\right)\right|-1\right) / 2\right\rceil$. This inequality is always an equality for the $C 3, C 4$ and $P 2$ operation.

To prove Theorem 1, the following lemma was used:

Lemma 4 (Farley and Proskurowski) If $G$ is an extremal immune graph not equal to $K_{1}$, then one of the operations $C 2, C 3, C 4$ or $P 2$ can be applied to $G$.

On this lemma our proof of Conjecture 2 is based.

\section{Overview of the proof}

The proof of Conjecture 2 is by contradiction, so first we assume an extremal immune graph exists that is not an ABC graph. Then we consider a graph with minimum number of vertices among all such graphs. This explains the following definition:

Definition 3 A graph $G$ is a minimum counterexample if it is extremal immune, it is not an ABC graph, and has minimum number of vertices among all such graphs.

We first consider the case that a minimum counterexample $G$ contains a $C_{2}$, so a $\mathrm{C} 2$ operation can be applied, resulting in vertex $v$. After applying this $\mathrm{C} 2$ operation, another extremal indecomposable graph $G^{\prime}$ is obtained (Lemma 3, Theorem 1). By our choice of $G, G^{\prime}$ must be an ABC graph. We consider a number of cases for $G^{\prime}$, for the choice of $v \in V\left(G^{\prime}\right)$ and for the possible graphs $G$ that can correspond to this, and in every case we obtain one of the following contradictions: $G$ has a matching-cut, $G$ is also an ABC graph, or a smaller counterexample exists.

If a minimum counterexample $G$ contains a triangle, we can apply operation $\mathrm{C} 3$ and find a contradiction in a similar way, and if $G$ contains a $C_{4}$, applying a $C 4$ operation leads to a contradiction. Finally, we can show that if a P2 operation can be applied resulting in the ABC graph $G^{\prime}$, then there is always a triangle or $C_{4}$ in $G^{\prime}$ that corresponds to a triangle or $C_{4}$ in $G$, so the previous cases can be applied. Since every operation leads to a contradiction, Lemma 4 shows that no counterexamples for the conjecture can exist.

\section{The structure of $A B C$ graphs}

Definition 4 A graph $G$ that can be obtained from a graph $H$ using only $B$ operations is called an $H$ component.

These graphs will often be used for $H$ in this case:

$K_{2}$ A $K_{2}$-component $G$ is also called an edge component. If $G$ can be constructed from a $K_{2}$ on vertices $u$ and $v$, then $G$ is called an edge component between $u$ and $v$.

$C_{3}$ A $C_{3}$-component is also called a triangle component. 
We can partition the edges of $\mathrm{ABC}$ graphs into edge induced components that are all $H$-components for $H=C_{3}, C_{2}$ or $P_{3}$, where $C_{3}$-components correspond to A operations in the decomposition, and the $\mathrm{C}$ operation corresponds to a $C_{2}$ or $P_{3}$-component:

Observation 5 For any decomposition of $A B C$ graph $G$ using $k$ A operations, we can partition the edges of $G$ into sets $A_{1}, \ldots, A_{k}$ and at most one set $C$ such that for every $i, G\left[A_{i}\right]$ is a triangle component, and $G[C]$ is a $C_{2}$ or $P_{3}$-component.

Observation 6 For any decomposition of an $H$-component $G$, we can partition $E(G)$ into sets $\left\{E_{u v}\right.$ : $u v \in E(H)\}$ such that $G\left[E_{u v}\right]$ is an edge component between $u$ and $v$. $u$ and $v$ are the only vertices of $G\left[E_{u v}\right]$ that can be incident with other edges in $G$.

Lemma 7 For every edge component $G$ between $u$ and $v$, and every edge e $\in E(G)$, a matching-cut $M$ exists that separates $u$ from $v$ with $e \in M$.

Lemma 8 If $u$ is a vertex in a triangle component $T$, then a decomposition of $T$ exists that starts on $u$.

\section{An example case}

Consider the case that minimum counterexample $G$ contains a triangle on vertices $u_{1}, u_{2}$ and $u_{3}$, and a $\mathrm{C} 3$ operation on this triangle yields $\mathrm{ABC}$ graph $G^{\prime}$ that consists of a single triangle component. Vertex $u \in V\left(G^{\prime}\right)$ corresponds to the contracted triangle.

Edges of $G^{\prime}$ correspond to edges of $G$, since the $\mathrm{C} 3$ operation consists of contraction and edge deletion operations. $G[M]$ denotes the graph induced by the edges of $G$ that correspond to $M \subset E\left(G^{\prime}\right)$ this way. If $G\left[E\left(G^{\prime}\right)\right]$ is isomorphic to $G^{\prime}$, then all edges incident with $u \in V\left(G^{\prime}\right)$ must w.l.o.g. be incident with $u_{1} \in V(G)$. In this case, $G$ can be obtained from $G^{\prime}$ with a single A operation on $u$ introducing $u_{2}$ and $u_{3}$, and renaming $u$ as $u_{1}$. So $G$ is an $\mathrm{ABC}$ graph in this case, a contradiction.

Now suppose $G\left[E\left(G^{\prime}\right)\right]$ is not isomorphic to $G^{\prime}$. By Lemma 8, we know that a decomposition of $G^{\prime}$ exists that starts with vertex $u$. Suppose the first A operation introduces vertices $v$ and $w$. Now the edges of $G^{\prime}$ can be partitioned into edge components $F_{u v}, F_{u w}$ and $F_{v w}$ that correspond to the edges introduced in this first A operation (Observation 6). Since $G\left[E\left(G^{\prime}\right)\right]$ is not isomorphic to $G^{\prime}$, it follows that there are edges $e \in E\left(F_{u v}\right)$ and $f \in E\left(F_{u w}\right)$ that are both incident with $u$ in $G^{\prime}$, but that correspond w.l.o.g. to edges incident with $u_{1}$ resp. $u_{2}$ in $G$. Since $G^{\prime}$ is a simple graph, $e$ and $f$ therefore correspond to non-adjacent edges in $G$. Now consider a matching-cut $M_{1}$ in $F_{u v}$ that separates $u$ from $v$, such that $e \in M_{1}$, and a matching-cut $M_{2}$ in $F_{u w}$ that separates $u$ from $w$, such that $f \in M_{2}$ (Lemma 7). Using Observation 6, it can be checked that $M_{1} \cup M_{2}$ corresponds to a matching-cut in $G$, which is a contradiction.

\section{Acknowledgements}

The author would like to thank Andrzej Proskurowski and Hajo Broersma for their suggestions and the discussions on this subject.

\section{References}

[Farley and Proskurowski(1984)] A. M. Farley and A. Proskurowski. Extremal graphs with no disconnecting matching. In Proceedings of the second West Coast conference on combinatorics, graph theory, and computing (Eugene, Ore., 1983), volume 41, pages 153-165, 1984. 